\title{
A Review Paper on OFDM and PAPR
}

\author{
Praveen Pawar ${ }^{1}$, Deepak Pancholi ${ }^{2}$ \\ ${ }^{1}$ PG Student ECE Department, LNCT, Indore, Madhya Pradesh, India \\ ${ }^{2}$ Assoc. Professor ECE Department, LNCT, Indore, Madhya Pradesh, India
}

\begin{abstract}
Orthogonal frequency-division multiplexing (OFDM) is a method of encoding digital data on multiple carrier frequencies. OFDM has developed into a popular scheme for wideband digital communication, whether wireless or over copper wires, used in applications such as digital television and audio Broadcasting, wireless networks, power line networks, and 4G mobile Communications. The transmit signals in an OFDM system can have high peak values in the time domain since many subcarrier components are added via an IFFT operation. Therefore, OFDM systems are known to have a high PAPR (Peak -to-Average Power Ratio), compared with single-carrier systems. In fact, the high PAPR is one of the most detrimental aspects in the OFDM system, as it decreases the SQNR (Signal -to-Quantization Noise Ratio) of ADC (Analog-to-Digital Converter) and DAC (Digital-to-Analog Converter) while degrading $g$ the efficiency of the power amplifier in the transmitter.
\end{abstract}

Keywords: PAPR, OFDM, IDFT, IFFT, ICI

\section{Peak-To-Average Power Ratio}

The PAPR is the relation between the maximum power of a sample in a given OFDM transmit symbol divided by the average power of that OFDM symbol. PAPR occurs when in a multicarrier system the different sub-carriers are out of phase with each other. At each instant they are different with respect to each other at different phase values. When all the points achieve the maximum value simultaneously; this will cause the output envelope to suddenly shoot up which causes a 'peak' in the output envelope. Due to presence of large number of independently modulated subcarriers in an OFDM system, the peak value of the system can be very high as compared to the average of the whole system. This ratio of the peak to average power value is termed as Peak-to Average Power Ratio.

As demand of high transmission rate is increasing day by day parallel transmission using multi-carrier is becoming a need of hour and OFDM is a promising candidate for various application such as Wireless Local area Networks (WLAN), Digital Video Broadcast (DVB), Digital Audio Broadcast (DAB), 4-G pertaining to its high band-width efficiency and its immunity towards ISI and delay spread [11], [12], [13]. OFDM avoids ISI problem by sending many low speed transmissions simultaneously with addition of cyclic prefix. However high peak to average power ratio is a major problem associated with OFDM system, this leads to increased complexity of analog to digital converter, digital to analog converter and reduced efficiency of the RF power amplifiers. The transmit signals in an OFDM system can have high peak values in the time domain since many subcarrier components are added via an IFFT operation. Therefore, OFDM systems are known to have a high PAPR (Peak-to-Average Power Ratio), compared with single-carrier systems [14], [15].

When high PAPR OFDM signal pass through a nonlinear device such high power amplifiers (HPA), it causes the out-of-band radiation that affects signals in adjacent bands, and in-band distortions that result in rotation, attenuation, and offset on the received signal. So a large back-off in input OFDM power is required to force the operation in linear region of HPA. Such HPA with large dynamic range are quite expensive and increase overall cost of the system. By reducing PAPR we reduce the overall cost as well as complexity of various components in the OFDM system.

The amount of PAPR reduction is proportional to the number of phase weighting factor. If the number of phase weighting factor is large, the number of parallel addition processor and the number of phase weighting factor sequences are searched to find the optimum combination of phase weighting factors will be increased incorporating huge complexity in the system. Then we cannot assume that the candidate signals are independent in PTS. The correlation among candidate signals deteriorates the PAPR reduction performance in PTS. The correlation among candidate signals is governed by two factors-one is the sub-block partition style as described in [6] and the other is the value of phase weighting factor set. So it is possible to alter these two factors to produce candidate signals with diminished correlation, so as to prevent degradation of the PAPR reduction performance.

\section{Introduction to OFDM}

Orthogonal frequency division multiplexing (OFDM) is a widely used modulation and multiplexing technology, which has become the basis of many telecommunications standards including wireless local area networks (LANs), digital terrestrial television (DTT) and digital radio broadcasting in much of the world[1].

In the past, as well as in the present, the OFDM is referred in the literature as Multi-carrier, Multi-tone and Fourier Transform. The OFDM concept is based on spreading the data to be transmitted over a large number of carriers, each being modulated at a low rate. The carriers are made orthogonal to each other by appropriately choosing the frequency spacing between them.

A multicarrier system, such as FDM divides the total available bandwidth in the spectrum into sub-bands for multiple carriers to transmit in parallel. It combines a large number of low data rate carriers to construct a composite high data rate communication system [2]. Orthogonality gives the carriers a valid reason to be closely spaced with

\section{Volume 5 Issue 6, June 2016} www.ijsr.net 


\section{International Journal of Science and Research (IJSR) \\ ISSN (Online): 2319-7064}

Index Copernicus Value (2013): 6.14 | Impact Factor (2015): 6.391

overlapping without ICI. With the increase of communications technology, the demand for higher data rate services such as multimedia, voice, and data over both wired and wireless links is also increased. New modulation schemes are required to transfer the large amount of data which existing techniques cannot support. These techniques must be able to provide high data rate, allowable Bit Error Rate (BER), and maximum delay. Orthogonal Frequency Division Multiplexing (OFDM) is one of them. OFDM has been used for Digital Audio Broadcasting (DAB) and Digital Video Broadcasting (DVB) in Europe and for Asymmetric Digital Subscriber Line (ADSL) high data rate wired links. OFDM has also been standardized as the physical layer for the wireless networking standard HIPERLAN2 in Europe and as the IEEE 802.11a, g standard in the US, promising raw data rates of between 6 and 54Mbps[3].

Orthogonal Frequency Division Multiplexing (OFDM) is a digital transmission Method developed to meet the increasing demand for higher data rates in communications which can be used in both wired and wireless environments.

\section{Advantages of OFDM}

OFDM has several advantages over single carrier modulation systems and these make it a viable alternative for CDMA in future wireless networks. In this section, I will discuss some of these advantages. OFDM is highly immune to multipath delay spread that causes inter-symbol interference in wireless channels. Since the symbol duration is made larger (by converting a high data rate signal into $\mathrm{N}$ low rate signals), the effect of delay spread is reduced by the same factor. Also by introducing the concepts of guard time and cyclic extension, the effects of inter-symbol interference (ISI) and inter-carrier interference (ICI) can be removed completely[20].

Immunity to frequency selective fading channels:

If the channel undergoes frequency selective fading, then complex equalization techniques are required at the receiver for single carrier modulation techniques. But in the case of OFDM the available bandwidth is split among many orthogonal narrowly spaced sub-carriers. Thus the available channel bandwidth is converted into many narrow flat- fading sub-channels. Hence it can be assumed that the subcarriers experience flat fading only, though the channel gain/phase associated with the sub-carriers may vary.

In the receiver, each sub-carrier just needs to be weighted according to the channel gain/phase encountered by it. Even if some sub-carriers are completely lost due to fading, proper coding and interleaving at the transmitter can recover the user data [20].

\section{PAPR Reduction Techniques}

Many methods have been suggested to reduce PAPR over the year [11]. PAPR reduction techniques vary according to the requirement of the system and are dependent on various factors such as PAPR Spectral efficiency, reduction capacity, increase in transmit signal power, loss in data rate, complexity of computation and increase in the bit-error rate (BER) at the receiver end are various factors which are taken into account before adopting a PAPR reduction technique of the system. Many techniques have been suggested for PAPR reduction, with different levels of success and complexity. Lot of techniques presents for the reduction of this PAPR [12] These techniques are divided into two groups - signal scrambling techniques and signal distortion techniques which are given below:

\section{Signal Scrambling Techniques}

- Block Coding Techniques

- Block Coding Scheme with Error Correction

- Selected Mapping (SLM)

- Partial Transmit Sequence (PTS)

- Interleaving Technique

- Tone Reservation (TR)

- Tone Injection (TI)

\section{Signal Distortion Techniques}

- Peak Windowing

- Envelope Scaling

- Peak Reduction Carrier

- Clipping and Filtering

\section{Conclusion}

The demand for high data rate wireless communication has been increasing drastically over the last decade. One way to transmit this high data rate information is to employ well known conventional single carrier systems. Since the transmission bandwidth is much larger than the coherence bandwidth of the channel, highly complex equalizers are needed at the receiver for accurately recovering the transmitted information.

The current implementations of OFDM do not fully exploit the capabilities of OFDM. There are still several avenues which can be explored to reduce the peak-topower ratio (PAPR) of OFDM signal. The PAPR performance of existing ICI cancellation schemes is either same or worse than normal OFDM signal. Therefore, the necessity to reduce the PAPR of normal OFDM signal and OFDM signal obtained from ICI cancellation schemes has been a prime motivating factor for this paper.

\section{References}

[1] G. Hill, M. Faulkner, and J. Singh, "Reducing the peak-to-average power ratio in OFDM by cyclically shifting partial transmit sequences," Electronics Letters, vol. 36, pp. 560-561, Mar 162000.

[2] Theory of Frequency Division Multiplexing: http://zone.ni.com/devzone/cda/ph/p/id/269

[3] Acosta, Guillermo. "OFDM Simulation Using MATLAB" 2000

[4] Litwin, Louis and Pugel, Michael. "The Principles of OFDM" 2001 
[5] S. Haene, A. Burg, D. Perels, P. Luethi, N. Felber, and W. Fichtner, "FPGA Implementation of Viterbi Decoders for MIMO-BICM," in Conference Record of the Thirty-Ninth Asilomar Conference on Signals, Systems and Computers, Oct. 28 - Nov. 012005.

[6] A. Jamin, and P. Mahonen, "Wavelet packet based modulation for wireless communications," Wiley Wireless Communications and Networking Journal, vol. 5, no. 2, pp. 123-137, Mar. 2005.

[7] F. S. Al-kamali , M. I. Dessouky, B. M. Sallam, F. E. Abd El-Samie , and F. Shawki, " An Efficient Transceiver scheme for SC-FDMA System Using A Wavelet Transform", IET Commun., 2010, Vol. 4, Iss. 1, pp. 69-79.

[8] F. Adachi, D. Garge, S. Takaoka, and K. Takeda, "Broadband CDMA techniques," IEEE Wireless Communs., Vol. 12, Issue 2, pp. 8-18, April 2005.

[9] X. Zhu, R.D. Murch, "Novel Frequency-Domain Equalization Architectures for a Single-carrier Wireless MIMO system", in proc. IEEE VTC, pp. 874-878, Fall 2002.

[10] Wu Y., Zou W. Y., "Orthogonal frequency division multiplexing: A multi-carrier modulation scheme", IEEE Trans. Consumer Electronics, vol. 41, no. 3, pp. 392-399, Aug. 1995.

[11] Van Nee R., Prasad R., "OFDM for Wireless Multimedia Communications", Artech House, 2000.

[12]Jiang T., Wu Y., "An Overview: Peak-to-average power ratio reduction techniques for FDM signals", IEEE Trans. Broadcasting, vol. 54, No. 2, pp. 257268, Jun. 2008.

[13] Armstrong, J., "Peak-to-average power reduction for OFDM by repeated clipping and frequency domain filtering," Electronics Letters, vol. 38, No. 5, pp. 246247, Feb. 2002.

[14] Wang X. B., Tjhung T. T., Ng C. S., "Reduction of peak-to-average power ratio of OFDM system using a companding technique", IEEE Transaction on Broadcasting, vol. 45, No. 3, pp. 303-307, Sept. 1999.

[15] Jiang T., Yang Y., Song Y., "Exponential companding transform for PAPR reduction in OFDM systems", IEEE Transaction on Broadcasting, vol. 51, No. 2, pp. 244-248, June 2005.

[16]Huang J. L., Zheng J., Letaief K. B., Gu J., "Companding Transform for Reduction in Peak-toAverage Power Ratio of OFDM Signals", IEEE Transaction on Wireless Communications, Vol. 03, No. 6, pp. 2030-2039, November 2004.

[17]Zhou, Y. , Jiang, T., “A Novel Multi-Point Square Mapping Combined with PTS to Reduce PAPR of OFDM Signals without Side Information", IEEE Transaction on Broadcasting, Vol. 55, No. 4, pp. 831835, Dec. 2009.

[18]Zhao Y., Häggman S. G., "Intercarrier interference self-cancellation scheme for OFDM mobile communication systems," IEEE Transaction on Communications, Vol. 49, No. 7, pp. 1185-1191, July 2001.

[19] Sathananthan K., Rajatheva, R. M. A. P., "Analysis of OFDM in the presence of Frequency Offset and a method to reduce performance degradation", Proc. of Global telecommunications conference'00, San Francisco, USA, Vol. 1 pp.72-76, Nov. 2000

Volume 5 Issue 6, June 2016 www.ijsr.net 